\title{
NIELSEN NUMBER IS A KNOT INVARIANT
}

\author{
ALEXANDER FEL'SHTYN \\ Instytut Matematyki, Uniwersytet Szczecinski \\ ul. Wielkopolska 15, 70-451 Szczecin, Poland \\ and \\ Boise State University \\ 1910 University Drive, Boise, Idaho, 83725-155, USA \\ E-mail: felshtyn@diamond.boisestate.edu,felshtyn@mpim-bonn.mpg.de
}

Abstract. We show that the Nielsen number is a knot invariant via representation variety.

1. Introduction. We briefly describe the few basic notions of Nielsen fixed point theory (see [2]). We assume $X$ to be a connected, compact polyhedron and $f: X \rightarrow X$ to be a continuous map. Let $p: \tilde{X} \rightarrow X$ be the universal cover of $X$ and $\tilde{f}: \tilde{X} \rightarrow \tilde{X}$ a lifting of $f$, i.e. $p \circ \tilde{f}=f \circ p$. Two liftings $\tilde{f}$ and $\tilde{f}^{\prime}$ are called conjugate if there is a $\gamma \in \Gamma \cong \pi_{1}(X)$ such that $\tilde{f}^{\prime}=\gamma \circ \tilde{f} \circ \gamma^{-1}$. The subset $p(\operatorname{Fix}(\tilde{f})) \subset \operatorname{Fix}(f)$ is called the fixed point class of $f$ determined by the lifting class $[\tilde{f}]$. Two fixed points $x_{0}$ and $x_{1}$ of $f$ belong to the same fixed point class iff there is a path $c$ from $x_{0}$ to $x_{1}$ such that $c \cong f \circ c$ (homotopy relative endpoints). This fact can be considered as an equivalent definition of a non-empty fixed point class. Every map $f$ has only finitely many non-empty fixed point classes, each a compact subset of $X$. A fixed point class is called essential if its index is non-zero. The number of essential fixed point classes is called the Nielsen number of $f$, denoted by $N(f)$. The Nielsen number is always finite and is a homotopy invariant. In the category of compact, connected polyhedra, the Nielsen number of a map is, apart from certain exceptional cases, equal to the least number of fixed points of maps with the same homotopy type as $f$.

Let us consider a braid representative of a knot and induced map of corresponding representation variety (see Section 2). We prove in Section 3 that the Nielsen number of induced map is invariant under Markov moves and so is a knot invariant.

The author came to the idea that Nielsen number is a knot invariant at the summer 2003, after conversations with Jochen Kroll and Uwe Kaiser. The author would like to thank the Max-Planck-Institute für Mathematik, Bonn, for kind hospitality and support.

2000 Mathematics Subject Classification: 37C25, 53D, 37C30, 55M20.

The paper is in final form and no version of it will be published elsewhere. 
2. Casson-Lin invariant. We recall firstly Lin's construction in [7] for the intersection number of the representation spaces corresponding to a braid representative of a knot $K$ in $S^{3}$. Let $\left(S^{3}, D_{+}^{3}, D_{-}^{3}, S^{2}\right)$ be a Heegard decomposition of $S^{3}$ with genus 0 , where

$$
S^{3}=D_{+}^{3} \cup_{S^{2}} D_{-}^{3}, \quad \partial D_{+}^{3}=\partial D_{-}^{3} D_{+}^{3} \cap D_{-}^{3}=S^{2} .
$$

Suppose that a knot $K \subset S^{3}$ is in general position with respect to this Heegard decomposition. So $K \cap S^{2}=\left\{x_{1}, \ldots, x_{n}, y_{1}, \ldots, y_{n}\right\}, K \cap D_{ \pm}^{3}$ is a collection of unknotted, unlinked $\operatorname{arcs}\left\{\gamma_{1}^{ \pm}, \ldots, \gamma_{n}^{ \pm}\right\} \subset D_{ \pm}^{3}$, where $\partial \gamma_{i}^{-}=\left\{x_{i}, y_{i}\right\}$ and $\left\{\gamma_{1}^{+}, \ldots, \gamma_{n}^{+}\right\}=K \cap D_{+}^{3}$ becomes a braid of $n$ strands inside $D_{+}^{3}$. Denote by $\beta$ a corresponding word in the braid group $B_{n}$. Given top end points $x_{i}$ of $\gamma_{i}^{+}$, the bottom end points of $\left\{\gamma_{1}^{+}, \ldots, \gamma_{n}^{+}\right\}$yield a permutation of $\left\{y_{1}, \ldots, y_{n}\right\}$ that gives us a map

$$
\pi: B_{n} \rightarrow S_{n}
$$

where $\pi(\beta)$ is the permutation of $\left\{y_{1}, \ldots, y_{n}\right\}$ in the symmetric group of $n$ letters. Let $K=\bar{\beta}$ be the closure of $\beta$. It is well-known that there is a correspondence between knots and braids $\beta$ for which $\pi(\beta)$ is a complete cycle of the $n$ letters (see [1]).

There is a corresponding Heegard decomposition for the complement of a $K$,

$$
\begin{gathered}
S^{3} \backslash K=\left(D_{+}^{3} \backslash K\right) \cup_{\left(S^{2} \backslash K\right)}\left(D_{-}^{3} \backslash K\right), \\
D_{ \pm}^{3} \backslash K=D_{ \pm}^{3} \backslash\left(D_{ \pm}^{3} \cap K\right), \quad S^{2} \backslash K=S^{2} \backslash\left(S^{2} \cap K\right) .
\end{gathered}
$$

Thus by Seifert-van Kampen theorem we have following diagram

$$
\begin{array}{cc}
\pi_{1}\left(S^{2} \backslash K\right) & \rightarrow \pi_{1}\left(D_{+}^{3} \backslash K\right) \\
\downarrow & \downarrow \\
\pi_{1}\left(D_{-}^{3} \backslash K\right) & \rightarrow \pi_{1}\left(S^{3} \backslash K\right),
\end{array}
$$

and a corresponding diagram of representation spaces

$$
\begin{array}{cc}
\mathcal{R}\left(S^{2} \backslash K\right) & \leftarrow \mathcal{R}\left(D_{+}^{3} \backslash K\right) \\
\uparrow & \\
\mathcal{R}\left(D_{-}^{3} \backslash K\right) & \leftarrow \mathcal{R}\left(S^{3} \backslash K\right),
\end{array}
$$

where $\mathcal{R}(X)=\operatorname{Hom}\left(\pi_{1}(X), S U(2)\right) / S U(2)$ for $X S^{2} \backslash K, D_{ \pm}^{3} \backslash K, S^{3} \backslash K$.

In [8], Magnus used the trace free matrices to represent the generators of a free group to show that the faithfulness of a representation of braid groups in the automorphism groups of the rings generated by the character functions on free groups. This is an original idea to have representations with trace free along all meridians which Lin worked in [7] to define the knot invariant. It has been carried out by M. Heusener and J. Kroll in [4] for the representation of knot groups with the trace of the meridian fixed (not necessary zero). Let $\mathcal{R}\left(S^{2} \backslash K\right)^{[i]}$ be the space of $S U(2)$ representations $\rho: \pi_{1}\left(S^{2} \backslash K\right) \rightarrow S U(2)$ such that

$$
\rho\left(\left[m_{x_{i}}\right]\right) \sim\left(\begin{array}{cc}
i & 0 \\
0 & -i
\end{array}\right), \quad \rho\left(\left[m_{y_{i}}\right]\right) \sim\left(\begin{array}{cc}
i & 0 \\
0 & -i
\end{array}\right),
$$

where $m_{x_{i}}, m_{y_{i}}, i=1,2, \ldots, n$, are the meridian circles around $x_{i}, y_{i}$ respectively. Note that $\pi_{1}\left(S^{2} \backslash K\right)$ is generated by $m_{x_{i}}, m_{y_{i}}, i=1,2, \ldots, n$, and one relation $\prod_{i=1}^{n} m_{x_{i}}=$ 
$\prod_{i=1}^{n} m_{y_{i}}$. Corresponding to (1), we have

$$
\begin{aligned}
& \mathcal{R}\left(S^{2} \backslash K\right)^{[i]} \leftarrow \mathcal{R}\left(D_{+}^{3} \backslash K\right)^{[i]} \\
& \uparrow \quad \uparrow \\
& \mathcal{R}\left(D_{-}^{3} \backslash K\right)^{[i]} \leftarrow \mathcal{R}\left(S^{3} \backslash K\right)^{[i]} .
\end{aligned}
$$

The conjugacy class in $S U(2)$ is completely determined by its trace. So the condition (2) can be reformulated for $\rho \in \mathcal{R}(X)^{[i]}$,

$$
\text { trace } \rho\left(\left[m_{x_{i}}\right]\right)=\operatorname{trace} \rho\left(\left[m_{y_{i}}\right]\right)=0 .
$$

The space $\mathcal{R}\left(S^{2} \backslash K\right)^{[i]}$ can be identified with the space of $2 n$ matrices $X_{1}, \ldots, X_{n}$, $Y_{1}, \ldots, Y_{n}$ in $S U(2)$ satisfying

$$
\begin{gathered}
\operatorname{trace}\left(X_{i}\right)=\operatorname{trace}\left(Y_{i}\right)=0 \quad \text { for } i=1, \ldots, n, \\
X_{1} \cdot X_{2} \cdots X_{n}=Y_{1} \cdot Y_{2} \cdots Y_{n} .
\end{gathered}
$$

Let $Q_{n}$ be the space $\left\{\left(X_{1}, \ldots, X_{n}\right) \in S U(2)^{n}\right.$ : $\left.\operatorname{trace}\left(X_{i}\right)=0, i=1, \ldots, n\right\}$. Let $\mathcal{R}^{*}\left(S^{2} \backslash K\right)^{[i]}$ be the subset of $\mathcal{R}\left(S^{2} \backslash K\right)^{[i]}$ consisting of irreducible representations. Note that $\mathcal{R}^{*}\left(S^{2} \backslash K\right)^{[i]}=\left(H_{n} \backslash S_{n}\right) / S U(2)$ in Lin's notation [7], where

$$
H_{n}=\left\{\left(X_{1}, \ldots, X_{n}, Y_{1}, \ldots, Y_{n}\right) \in Q_{n} \times Q_{n}: X_{1} \cdots X_{n}=Y_{1} \cdots Y_{n}\right\},
$$

$S_{n}$ is the subspace of $H_{n}$ consisting of all the reducible points. Here $H_{n} \backslash S_{n}$ is the total space of a $S U(2)$-fiber bundle over $\mathcal{R}^{*}\left(S^{2} \backslash K\right)^{[i]}$.

Given $\beta \in B_{n}$, we denote by $\Gamma_{\beta}$ the graph of $\beta$ in $Q_{n} \times Q_{n}$, i.e.

$$
\Gamma_{\beta}=\left\{\left(X_{1}, \ldots, X_{n}, \beta\left(X_{1}\right), \ldots, \beta\left(X_{n}\right)\right) \in Q_{n} \times Q_{n}\right\} .
$$

As an automorphism of the free group $Z\left[m_{x_{1}}\right] * Z\left[m_{x_{2}}\right] * \cdots * Z\left[m_{x_{n}}\right]$, this element $\beta \in B_{n}$ preserves the word $\left[m_{x_{1}}\right] \cdots\left[m_{x_{n}}\right]$. Thus we have $X_{1} \cdots X_{n}=\beta\left(X_{1}\right) \cdots \beta\left(X_{n}\right)$, or in other words $\Gamma_{\beta}$ is a subspace of $H_{n}$. In fact, for $\bar{\beta}=K$, this subspace $\Gamma_{\beta}$ coincides with the subspace of representations $\rho: \pi_{1}\left(S^{2} \backslash K\right) \rightarrow S U(2)$ in $H_{n}$ which can be extended to $\pi_{1}\left(D_{+}^{3} \backslash K\right), \Gamma_{\beta}=\operatorname{Hom}\left(\pi_{1}\left(D_{+}^{3} \backslash K\right), S U(2)\right)^{[i]}$. Hence the space $\mathcal{R}^{*}\left(D_{+}^{3} \backslash K\right)^{[i]}=\Gamma_{\beta, \text { irre }} / S U(2)$ is the irreducible $S U(2)$ representations with traceless condition over $D_{+}^{3} \backslash K$.

In the special case $\beta=$ id, $\Gamma_{\text {id }}$ represents the diagonal in $Q_{n} \times Q_{n}$,

$$
\Gamma_{\text {id }}=\left\{\left(X_{1}, \ldots, X_{n}, X_{1}, \ldots, X_{n}\right) \in Q_{n} \times Q_{n}\right\} .
$$

Since $K \cap D_{-}^{3}$ represents the trivial braid, this space $\Gamma_{\text {id }} \subset H_{n}$ can be identified with the subspace of representations in $\operatorname{Hom}\left(\pi_{1}\left(S^{2} \backslash K\right), S U(2)\right)^{[i]}$ which can be extended to $\pi_{1}\left(D_{-}^{3} \backslash K\right)$, i.e. $\Gamma_{\text {id }}=\operatorname{Hom}\left(\pi_{1}\left(D_{-}^{3} \backslash K\right), S U(2)\right)^{[i]}$. By Seifert-van Kampen Theorem, the intersection $\Gamma_{\beta} \cap \Gamma_{\text {id }}$ is the same as the space of representations of $\pi_{1}\left(S^{3} \backslash K\right)$ satisfying the monodromy condition $[i]$ (see (1)),

$$
\Gamma_{\beta} \cap \Gamma_{\mathrm{id}}=\operatorname{Hom}\left(\pi_{1}\left(S^{3} \backslash K\right), S U(2)\right)^{[i]} .
$$

Given $\beta \in B_{n}$ with $\bar{\beta}=K$, there is an induced diffeomorphism (still denoted by $\beta$ ) from $Q_{n}$ to itself. Such a diffeomorphism also induces a diffeomorphism

$$
f_{\beta}: \mathcal{R}^{*}\left(S^{2} \backslash K\right)^{[i]} \rightarrow \mathcal{R}^{*}\left(S^{2} \backslash K\right)^{[i]}
$$

of the representation variety. 
Note that $\bar{\Gamma}_{\beta}=\left(\Gamma_{\beta} \backslash\left(\Gamma_{\beta} \cap S_{n}\right)\right) / S U(2)$ is the image of the "diagonal" $\bar{\Gamma}_{\text {id }}$ under diffeomorphism $f_{\beta}$. By Seifert-van Kampen theorem (3), it is clear that the fixed point set of $f_{\beta}$ is

$$
\operatorname{Fix}\left(\left.f_{\beta}\right|_{\mathcal{R}^{*}\left(S^{2} \backslash K\right)^{[i]}}\right) \bar{\Gamma}_{\beta} \cap \bar{\Gamma}_{\text {id }} \mathcal{R}^{*}\left(S^{3} \backslash K\right)^{[i]} .
$$

The oriented submanifolds $\bar{\Gamma}_{\beta}=\mathcal{R}^{*}\left(D_{+}^{3} \backslash K\right)^{[i]}, \bar{\Gamma}_{\text {id }}=\mathcal{R}^{*}\left(D_{-}^{3} \backslash K\right)^{[i]}$ intersect each other in a compact subspace of $\mathcal{R}^{*}\left(S^{2} \backslash K\right)^{[i]}$ by Lemma 1.6 from [7]. Hence we can perturb $f_{\beta}$, or in other words, perturb $\mathcal{R}^{*}\left(D_{+}^{3} \backslash K\right)^{[i]}$ to $\hat{\mathcal{R}}^{*}\left(D_{+}^{3} \backslash K\right)^{[i]}$ by a compactly support isotopy so that $\hat{\mathcal{R}}^{*}\left(D_{+}^{3} \backslash K\right)^{[i]}$ intersects $\mathcal{R}^{*}\left(D_{-}^{3} \backslash K\right)^{[i]}$ transversally at a finite number of intersection points. Denote the perturbed diffeomorphism by $\hat{f}_{\beta}$. So its fixed points are all non-degenerated.

The Casson-Lin invariant of a knot $K=\bar{\beta}$ is given by counting the algebraic intersection number of $\hat{\mathcal{R}}^{*}\left(D_{+}^{3} \backslash K\right)^{[i]}$ and $\mathcal{R}^{*}\left(D_{-}^{3} \backslash K\right)^{[i]}$, or the algebraic number of $\operatorname{Fix}\left(\hat{f}_{\beta}\right)$,

$$
\begin{aligned}
\lambda_{C L}(K)=\lambda_{C L}(\beta)=\operatorname{Algebraic}(\# & \left.\operatorname{Fix}\left(\hat{f}_{\beta}\right)\right) \\
& =\operatorname{Algebraic}\left(\#\left(\hat{\mathcal{R}}^{*}\left(D_{+}^{3} \backslash K\right)^{[i]} \cap \mathcal{R}^{*}\left(D_{-}^{3} \backslash K\right)^{[i]}\right)\right) .
\end{aligned}
$$

The results proved by Lin in [7] show that the Casson-Lin invariant $\lambda_{C L}(K)=\lambda_{C L}(\beta)$ is independent of its braid representatives, i.e. $\lambda_{C L}(\beta)$ is invariant under the Markov moves of type I and type II on $\beta$ and is one half of the classical signature of the knot $K$.

3. Nielsen number is a knot invariant. In this section we propose to count fixed points of $f_{\beta}$ in a Nielsen way, namely, using the classical Nielsen numbers of $f_{\beta}$. Nielsen counting of fixed points is a counting in the presence of the fundamental group. In order to get an invariant of knots from braids, we have to verify that Nielsen number $N\left(f_{\beta}\right)$ is invariant under Markov moves. A Markov move of type I changes $\sigma \in B_{n}$ to $\xi^{-1} \sigma \xi \in B_{n}$ for any $\xi \in B_{n}$, and the Markov move of type II changes $\sigma \in B_{n}$ to $\sigma_{n}^{ \pm} \sigma \in B_{n+1}$, or the inverses of these operations. It is well-known that two braids $\beta_{1}$ and $\beta_{2}$ have isotopic closure if and only if $\beta_{1}$ can be changed to $\beta_{2}$ by a sequence of finitely many Markov moves [1].

TheOREM 1. If $\overline{\beta_{1}}=\overline{\beta_{2}}=K$ is a knot, $\beta_{1} \in B_{n}, \beta_{2} \in B_{m}$, then

$$
N\left(f_{\beta_{1}}\right)=N\left(f_{\beta_{2}}\right) \text {. }
$$

So the Nielsen number $N\left(f_{\beta}\right)$ is a knot invariant.

Proof. We only need to show that for $\beta \in B_{n}$ with $\bar{\beta}$ being a knot $K$, the Markov moves of type I and type II on $\beta$ provide either a conjugacy or a isotopy of $f_{\beta}$. Hence from the commutativity and the invariance property under isotopy of the Nielsen numbers, we get that $N\left(f_{\beta}\right)$ is an invariant of knot $K=\bar{\beta}$.

Suppose we have the Markov move of type I: change $\beta$ to $\xi^{-1} \beta \xi$ for some $\xi \in B_{n}$. The element $\xi$ in $B_{n}$ induces a diffeomorphism $\xi: Q_{n} \rightarrow Q_{n}$, which is orientation preserving as observed by $\operatorname{Lin}$ in [7]. Note that $B_{n}$ is generated by $\sigma_{1}, \ldots, \sigma_{n-1}$. For any $\sigma_{i}^{ \pm}$, the induced diffeomorphism $\sigma_{i}^{ \pm} \times \sigma_{i}^{ \pm}: Q_{n} \times Q_{n} \rightarrow Q_{n} \times Q_{n}$ is an orientation preserving diffeomorphism. So $\xi$ is also a orientation preserving diffeomorphism since orientation preserving properties are invariant under the composition operation. Hence there is a 
homeomorphism

$$
\xi \times \xi: Q_{n} \times Q_{n} \rightarrow Q_{n} \times Q_{n},
$$

which commutes with the $S U(2)$-action and

$$
\begin{aligned}
(\xi \times \xi)\left(\mathcal{R}^{*}\left(S^{2} \backslash K\right)^{[i]}\right)=\mathcal{R}^{*}\left(S^{2} \backslash K\right)^{[i]} & (\text { changing variables by } \xi \times \xi), \\
(\xi \times \xi)\left(\mathcal{R}^{*}\left(D_{-}^{3} \backslash K\right)^{[i]}\right)=\mathcal{R}^{*}\left(D_{-}^{3} \backslash K\right)^{[i]} & \text { (in new coordinate } \left.\xi\left(X_{1}\right), \ldots, \xi\left(X_{n}\right)\right), \\
(\xi \times \xi)\left(\mathcal{R}^{*}\left(D_{+}^{3} \backslash K\right)^{[i]}\right)=\mathcal{R}^{*}\left(D_{+}^{3} \backslash K\right)^{[i]} & \text { (in new coordinate } \left.\xi\left(X_{1}\right), \ldots, \xi\left(X_{n}\right)\right),
\end{aligned}
$$

as oriented manifolds. Let $g_{\xi}: \mathcal{R}^{*}\left(S^{2} \backslash K\right)^{[i]} \rightarrow \mathcal{R}^{*}\left(S^{2} \backslash K\right)^{[i]}$ be the induced homeomorphism, induced from $\xi \times \xi$ as coordinate changes. Hence we get a conjugacy relation

$$
g_{\xi}^{-1} \circ f_{\beta} \circ g_{\xi}=f_{\xi^{-1} \beta \xi},
$$

from changing variables via $g_{\xi}$. Note that $\operatorname{Fix}\left(f_{\xi^{-1} \beta \xi}\right)$ is identified with $\operatorname{Fix}\left(f_{\beta}\right)$ under $g_{\xi}$. Thus the Markov move of type I preserves the conjugacy class of $f_{\beta}$. Therefore by commutativity of the Nielsen number (see [5]) we have

$$
N\left(f_{\xi^{-1} \beta \xi}\right) N\left(g_{\xi}^{-1} \circ f_{\beta} \circ g_{\xi}\right)=N\left(f_{\beta}\right) .
$$

It is clear that the argument goes through for the inverse operation of Markov move of type I.

Suppose we have the Markov move of type II: change $\beta$ to $\sigma_{n} \beta \in B_{n+1}$. Recall that $\sigma_{n}\left(x_{i}\right)=x_{i}, 1 \leq i \leq n-1, \sigma_{n}\left(x_{n}\right)=x_{n} x_{n+1} x_{n}^{-1}$ and $\sigma_{n}\left(x_{n+1}\right)=x_{n}$. We need to identify the Nielsen number from the construction in $\hat{H}_{n}$ into the one from $\hat{H}_{n+1}$. Following Lin [7], there is an imbedding $q: Q_{n} \times Q_{n} \rightarrow Q_{n+1} \times Q_{n+1}$ given by

$$
q\left(X_{1}, \ldots, X_{n}, Y_{1}, \ldots, Y_{n}\right)=\left(X_{1}, \ldots, X_{n}, Y_{n}, Y_{1}, \ldots, Y_{n}, Y_{n}\right) .
$$

Such an imbedding commutes with the $S U(2)$-action and $q\left(H_{n}\right) \subset H_{n+1}$, and induces an imbedding

$$
\hat{q}: \hat{H}_{n}\left(=\mathcal{R}^{*}\left(S^{2} \backslash \bar{\beta}\right)^{[i]}\right) \rightarrow \hat{H}_{n+1}\left(=\mathcal{R}^{*}\left(S^{2} \backslash \overline{\sigma_{n} \beta}\right)^{[i]}\right) .
$$

Note that the symplectic structure of $\hat{H}_{n+1}$ restricted on $\hat{q}\left(\hat{H}_{n}\right)$ is the symplectic structure on $\hat{H}_{n}$. Under this imbedding, we see that $\hat{q}\left(f_{\beta}\right): \hat{H}_{n+1} \rightarrow \hat{H}_{n+1}$ is given by

$$
\left(X_{1}, \ldots, X_{n}, X_{1}, \ldots, X_{n}\right) \mapsto\left(X_{1}, \ldots, X_{n}, \beta\left(X_{n}\right), \beta\left(X_{1}\right), \ldots, \beta\left(X_{n}\right), \beta\left(X_{n}\right)\right) .
$$

The image of $\hat{q}\left(f_{\beta}\right)$ is invariant under the operation of $\sigma_{n}$. Also the corresponding diffeomorphism $f_{\sigma_{n} \beta}$ is given by

$$
\begin{aligned}
f_{\sigma_{n} \beta}\left(X_{1}, \ldots,\right. & \left.X_{n}, X_{n+1}, X_{1}, \ldots, X_{n}, X_{n+1}\right) \\
& =\left(X_{1}, \ldots, X_{n+1}, \beta\left(X_{1}\right), \ldots, \beta\left(X_{n-1}\right), \beta\left(X_{n}\right) X_{n+1} \beta\left(X_{n}\right)^{-1}, \beta\left(X_{n}\right)\right) .
\end{aligned}
$$

Thus we have

$$
\hat{q}\left(\mathcal{R}^{*}\left(D_{-}^{3} \backslash \bar{\beta}\right)^{[i]}\right) \subset \mathcal{R}^{*}\left(D_{-}^{3} \backslash \overline{\sigma_{n} \beta}\right)^{[i]}, \quad \hat{q}\left(\mathcal{R}^{*}\left(D_{+}^{3} \backslash \bar{\beta}\right)^{[i]}\right) \subset \mathcal{R}^{*}\left(D_{+}^{3} \backslash \overline{\sigma_{n} \beta}\right)^{[i]} .
$$

The fixed points of $f_{\sigma_{n} \beta}$ are elements

$$
\beta\left(X_{i}\right)=X_{i}, 1 \leq i \leq n_{1} ; \quad \beta\left(X_{n}\right) X_{n+1} \beta\left(X_{n}\right)^{-1}=X_{n}, \quad \beta\left(X_{n}\right)=X_{n+1},
$$

which is equivalent to $\beta\left(X_{i}\right)=X_{i}, 1 \leq i \leq n$, i.e.

$$
\operatorname{Fix}\left(f_{\sigma_{n} \beta}\right)=\operatorname{Fix}\left(\hat{q}\left(f_{\beta}\right)\right)=\operatorname{Fix}\left(f_{\beta}\right) .
$$


Then there is a (Hamiltonian) isotopy $\psi_{t}: \hat{H}_{n+1}\left(=\left(H_{n+1} \backslash S_{n+1}\right) / S U(2)\right) \rightarrow \hat{H}_{n+1}$ between $\psi_{t_{0}}=\hat{q}\left(f_{\beta}\right)$ by $(8)$ and $\psi_{t_{1}}=f_{\sigma_{n} \beta}(9)$ (see $[6,7]$ for the explicit constructions). So we have

$$
N\left(f_{\sigma_{n} \beta}\right)=N\left(\hat{g}\left(f_{\beta}\right)\right) N\left(f_{\beta}\right) .
$$

The first equality follows from the invariance property of Nielsen numbers under the isotopy $\psi_{t}$ and the second from the natural identification. We can similarly prove that

$$
N\left(f_{\sigma_{n}^{-1} \beta}\right) N\left(f_{\beta}\right) \text {. }
$$

REMARK 2. It is known for a long time that the problem of computation of Nielsen numbers is a very difficult problem. By this reason, we strongly believe that the Nielsen number $N\left(f_{\beta}\right)$ is a new knot invariant, which cannot be reduced to the known knot invariants, as it happened in the case of the Casson-Lin invariant of knots.

Example 3 ("Pillowcase"). For $n=2$ the irreducible variety $\mathcal{R}^{*}\left(S^{2} \backslash K\right)^{[i]}$ is a 2-sphere with four cone points deleted (see [7]). So, in this case the space $\mathcal{R}^{*}\left(S^{2} \backslash K\right)^{[i]}$ is nonsimply-connected and the Nielsen number $N\left(f_{\beta}\right)$ is not trivial for general $\beta$.

Question 4. Is the space $\mathcal{R}^{*}\left(S^{2} \backslash K\right)^{[i]}$ simply-connected if $n>2$ ?

The author was informed by Hans Boden that Theorem 8.3 in [3] about a moduli space of stable parabolic bundles over 2-sphere with marked points with given degree and weights may be very useful for the full answer on this question.

\section{References}

[1] J. S. Birman, Braids, links and mapping class groups, Ann. of Math. Stud. 82, Princeton Univ. Press, Princeton 1974.

[2] A. L. Fel'shtyn, Dynamical zeta functions, Nielsen theory and Reidemeister torsion, Mem. Amer. Math. Soc. 147 (2000), no. 699.

[3] M. Furuta, B. Steer, Seifert fibred homology 3-spheres and Yang-Mills equations on Riemann surfaces with marked points, Adv. Math. 96 (1992), 38-102.

[4] M. Heusener, J. Kroll, Deforming abelian SU(2)-representations of knot groups, Comment. Math. Helv. 73 (1998), 480-498.

[5] B. Jiang, Lectures on Nielsen Fixed Point Theory, Contemp. Math. 14, Amer. Math. Soc., Providence 1983.

[6] W. Li, Casson-Lin's invariant and Floer homology, J. Knot Theory Ramifications 6 (1997), 851-877.

[7] X. S. Lin, A knot invariant via representation spaces, J. Differential Geom. 35 (1992), $337-357$.

[8] W. Magnus, Rings of Fricke characters and automorphism groups of free groups, Math. Z. 170 (1980), 91-103. 